\title{
Endothelial function progressively deteriorates during normal pregnancy.
}

\section{$\operatorname{AUTHOR}(\mathrm{S})$ :}

Fujita, Kohei; Tatsumi, Keiji; Kondoh, Eiji; Chigusa, Yoshitsugu; Mogami, Haruta; Fujita, Masatoshi; Konishi, Ikuo

\section{CITATION:}

Fujita, Kohei ...[et al]. Endothelial function progressively deteriorates during normal pregnancy.. Hypertension in pregnancy 2013, 32(2): 129138

\section{ISSUE DATE:}

2013-05

URL:

http://hdl.handle.net/2433/189762

\section{RIGHT:}

(c) 2013 Informa Healthcare.; This is not the published version. Please cite only the published version.; この論文は出版社版でありません。引 用の際には出版社版をご確認ご利用ください。 
Endothelial Function in Pregnancy

\section{Endothelial Function Progressively Deteriorates During Normal Pregnancy}

3 Kohei Fujita, ${ }^{1}$ Keiji Tatsumi, ${ }^{1}$ Eiji Kondoh, ${ }^{1}$ Yoshitsugu Chigusa, ${ }^{1}$ Haruta Mogami, ${ }^{1}$

4 Masatoshi Fujita, $^{2}$ Ikuo Konishi $^{1}$

$6{ }^{1}$ Department of Gynecology and Obstetrics, Kyoto University Graduate School of Medicine,

7 54 Shogoin-Kawaharacho, Sakyo-ku, Kyoto, Japan

$8 \quad{ }^{2}$ Department of Cardiology, Uji Hospital, 54-2 Gokasho, Shibanohigashi, Uji, Kyoto, Japan

10 Corresponding author: Kohei Fujita, M.D., Ph.D.

11 Department of Gynecology and Obstetrics

12 Kyoto University Graduate School of Medicine

1354 Shogoin-Kawaharacho, Sakyo-ku, Kyoto 606-8507, Japan

$14 \quad$ Tel.: $+81-75-751-3269$

15 Fax: +81-75-761-3967

16 E-mail: gynef@kuhp.kyoto-u.ac.jp 
Endothelial Function in Pregnancy

\section{ABSTRACT}

2 Objective. To elucidate changes in endothelial function throughout the gestational period in

3 normal pregnancy and its relationship with plasma sFlt-1 levels. Methods. Endothelial

4 function was evaluated by reactive hyperemia index (RHI) using Endo-PAT2000 and plasma

5 sFlt-1 levels were measured simultaneously by ELISA. Results. RHI gradually deteriorated

6 with increasing the gestational age. Plasma sFlt-1 levels exhibited a gradual increase at late

7 pregnancy and were inversely correlated with RHI. Conclusion. Maternal endothelial function

8 gradually deteriorates with increasing gestational age and there is an inverse correlation

9 between endothelial function and plasma sFlt-1 levels in normal pregnancy.

11 Keywords Peripheral arterial tonometry, Pregnancy, Endothelial dysfunction, sFlt-1,

12 Preeclampsia. 
Endothelial Function in Pregnancy

\section{INTRODUCTION}

2 In normal pregnancy, blood volume and cardiac output increase as pregnancy proceeds. By

3 contrast, blood pressure gradually decreases slightly toward mid-pregnancy and then slowly

4 increases toward the term (1). It is assumed that this discrepancy is due at least in part to

5 alterations in endothelial function based on reductions in systemic vascular resistance by

6 nitric oxide (NO) (2). In preeclampsia (PE), it is acknowledged that poor placentation in early

7 pregnancy causes placental hypoperfusion with subsequent maternal endothelial dysfunction.

8 The endothelial dysfunction in various tissues is implicated in symptoms such as hypertension,

9 proteinuria, tissue oedema, cerebral haemorrhage, and so on (3). Therefore an assessment of

10 endothelial function might be useful for prediction, prevention, and curative strategies for PE

11 patients. However, it is still controversial whether endothelial function changes throughout

12 normal pregnancy (4-8).

13 Endothelial function includes vasodilatory, anti-coagulant, anti-inflammatory and

14 vascular permeability activities. It is thought that vascular homeostasis is well maintained (9)

15 and NO and vascular endothelial growth factor (VEGF) play central roles in these

mechanisms. Soluble fms-like tyrosine kinase-1 (sFlt-1) has been implicated in the symptoms

17 of PE. It is a soluble type of VEGFR-1 and competes with VEGF for binding to VEGF receptors. Rats over-expressing sFlt-1 show similar clinical features of PE (10). In PE patients, 
Endothelial Function in Pregnancy

$1 \quad(10,11)$ and the importance of elevated sFlt-1 levels has been discussed in relation to

2 endothelial dysfunction (12). However, the relationship between circulating sFlt-1 levels and

3 endothelial function in normal pregnancy remains unclear.

4 To assess endothelial function, flow-mediated dilation (FMD) of the brachial artery

5 during reactive hyperemia $(\mathrm{RH})$ has been introduced. Though FMD is a gold standard for

6 non-invasive assessment of endothelial function, the measurement of FMD has a potential

7 limitation regarding its intra-and inter-operator reproducibility (13). To overcome this

8 problem, peripheral arterial tonometry (PAT) has recently been developed. The Endo-

9 PAT2000 system (Itamar Medical, Caesarea, Israel) does not require the operator to have the

10 training and specialized skill needed when using ultrasonography. Corretti reported that PAT

11 method is significantly correlated with FMD $(p<0.0001)(13)$. In comparing PAT with FMD,

12 Carty measured PAT and FMD in the same pregnant women. In the result, it was showed that

13 both parameters compared favorably (14). The data reflect arterial tone alterations in the

14 peripheral microvascular bed and can be digitalized and read as reactive hyperemia index

15 (RHI). The influence of the autonomic nervous system can be eliminated by measuring the

16 contralateral arm as a control. Thus, the PAT method might be superior to FMD in terms of

17 convenience, quantitation, and reproducibility (15). RHI in patients with coronary endothelial

18 dysfunction was reported to be relatively lower (16). Another study demonstrated that

19 administration of L-NAME, known as an inhibitor of eNOS, reduced RHI, implicating NO- 
Endothelial Function in Pregnancy

1 dependent endothelial function (17). In addition, Aragones reported that elevated levels of sE-

2 selectin, a biomarker for endothelial dysfunction, were associated with lower RHI (18).

3 However there have been few articles so far investigating the role of endothelial function

4 using RHI in PE patients. One report presented reduced RHI in patients with PE compared

5 with normal pregnant women around 32 weeks of gestation (19). Recently, Carty et al.

6 demonstrated that RHI in pregnant women at 28 weeks was significantly lower than that at 16

7 weeks (14), but they just focused on the predictive value of the onset of PE and concluded

8 that it failed for that purpose.

9 Here we report a prospective study of monitoring maternal RHI to evaluate endothelial

10 function throughout the normal gestational period using the Endo-PAT system. We also

11 measured endothelial function and circulating sFlt-1 levels in non-pregnant women,

12 preeclamptic patients and pregnant women who develop PE later. Because the significance of

13 increased sFlt-1 levels in normal late gestation has not been clarified, we measured plasma

14 sFlt-1 levels in all subjects and evaluated the relationship between RHI and plasma sFlt-1

15 levels. The aim of this study was to elucidate alterations in endothelial function using the PAT

16 system and its correlation to plasma sFlt-1 levels throughout the normal gestational period as

17 well as the process of PE. 
Endothelial Function in Pregnancy

\section{MATERIALS AND METHODS}

\section{Study Population}

All pregnant women consulting our department at Kyoto University Hospital between

4 March 2011 and March 2012 were considered for enrolment. Complicated cases with chronic

5 hypertension, diabetes mellitus, chronic kidney disease, autoimmune diseases like systemic

6 lupus erythematosus and respiratory diseases were excluded. Over eighty percent of eligible

7 pregnant women were enrolled in the present study. Age-matched, apparently healthy non-

8 pregnant women were also enrolled. In addition, we enrolled 7 patients who had already

9 developed symptoms of PE and had been treated with antihypertensive drugs. The protocol

10 was approved by the institutional ethics committee of Kyoto University Graduate School of

11 Medicine. Written informed consent was obtained from all subjects.

Measurement of Endothelial Function

PAT is a new technique to non-invasively assess endothelial function. It comprises

finger probes to evaluate digital volume changes accompanied by pulse waves (20). We used

the Endo-PAT2000 system to evaluate endothelial function. All participants were asked to 
Endothelial Function in Pregnancy

1 controlled $\left(23\right.$ to $\left.26{ }^{\circ} \mathrm{C}\right)$, quiet and dark room was reserved for the examination. A blood

2 pressure cuff was placed on one upper arm and a probe was placed on the tip of the index

3 finger; the probe sensor on the contralateral arm served as a control. Following approximately

415 min of resting in a supine position on the bed, the baseline digital volume signals were

5 recorded for $5 \mathrm{~min}$, then the blood pressure cuff was inflated to $200 \mathrm{mmHg}$ to occlude the

6 brachial artery and the pressure was maintained for $5 \mathrm{~min}$. The cuff was then released to

7 induce $\mathrm{RH}$ and the reaction was recorded for $5 \mathrm{~min}$. The analytical method of calculating RHI

8 was as follows. The RHI reflects the ratio of the alteration in the amplitude from 90 to 150

9 seconds after the cuff is released (occluded arm -A; non-occluded arm - C) divided by the

10 average amplitude of the signal from 170 to 20 seconds before the cuff is inflated (occluded

11 arm - B; non-occluded arm - D). This value was calculated using the formula: $\mathrm{RHI}=(\mathrm{A} / \mathrm{B})$ /

12 (C/D). The RHI data were digitally analysed using the Endo-PAT2000 software version 3.3.2

13 (Itamar Medical). Each pregnant woman underwent this test 1 to 3 times at intervals of longer

14 than one month apart.

\section{Plasma Sampling and Measurement of sFlt-1 Levels}

Plasma samples were collected from all pregnant women who participated in this study 
Endothelial Function in Pregnancy

1 centrifugation at $3000 \mathrm{rpm}$ for $30 \mathrm{~min}$. The supernatants were stored at $-20{ }^{\circ} \mathrm{C}$ until analysis.

2 Plasma sFlt-1 levels were measured using a human sVEGFR1/Flt-1 enzyme-linked

3 immunosorbent assay (ELISA) kit (R\&D Systems, Minneapolis, MN) in duplicate according

4 to the manufacturer's protocol. Inter-assay and intra-assay coefficients of variation were 5.5-

$5 \quad 9.8 \%$ and $2.6-3.8 \%$, respectively.

6

7 Diagnosis of $\mathbf{P E}$

PE was defined as the new onset of hypertension and proteinuria after 20 weeks of

9 gestation. Hypertension was defined as a systolic blood pressure $\geq 140 \mathrm{mmHg}$ or diastolic

10 blood pressure $\geq 90 \mathrm{mmHg}$ on two measurements at least 4 hours apart, and proteinuria as $\geq$

$11300 \mathrm{mg} /$ day (21). In Japan, it is defined that late-onset preeclampsia develops at or after 32

12 weeks of gestation.

14 Statistical Analysis

The results of normally distributed continuous variables are expressed as the mean \pm

SEM (range), while those with skewed distribution are expressed as the median value with

17 [interquartile range]. Continuous variables were analysed by the Wilcoxon $t$ test, Mann- 
Endothelial Function in Pregnancy

1 Statistical analyses were performed using Prism 3.0 (GraphPad Software, La Jolla, CA).

\section{RESULTS}

\section{Patient Characteristics}

The information collected on the study population is shown in Table 1 . Three of 29

pregnant women developed PE. Body mass indexes were significantly higher in the normal

7 control pregnant women than in the non-pregnant women. In 7 PE patients, systolic and

8 diastolic blood pressures were not high due to antihypertensive treatment and the median time

9 of measuring RHI was 32 [31.5-34] weeks of gestation. The average of systolic blood

10 pressure was $161 \pm 5 \mathrm{mmHg}$, and the diastolic blood pressure was $95 \pm 4 \mathrm{mmHg}$, when PE

11 patients were diagnosed at first time. RHI measurements in PE patients were performed 4

12 [2.5-5.5] days after start of the treatment. Gestational age at delivery was earlier in the PE

13 group than in normal pregnant women. Neonatal weight was also lighter in the PE patients

14 than in normal pregnant women and patients with late PE development.

\section{Alterations of RHI in Normal Pregnancy}

The RHI gradually decreased throughout gestation in normal pregnant women (Figure 
Endothelial Function in Pregnancy

1 between non-pregnant women and pregnant women under 19 weeks of gestation (Figure 1B).

2

\section{Relation of RHI to Plasma sFlt-1 Levels}

Plasma sFlt-1 levels gradually increased throughout gestation in normal pregnant women

(Figure 2A). They were higher in pregnant women over 32 weeks of gestation than those at earlier periods of gestation (Figure 2B). The RHI was inversely correlated with plasma sFlt-1 levels $(p=0.0074)$ for all 56 measurements in normal pregnant women (Figure 3).

Figure 4 shows comparisons of RHI (A) and plasma sFlt-1 levels (B) during 3 gestational periods ( $\leq 19$ weeks, $20-31$ weeks, $\geq 32$ weeks). Nine normal pregnant women were given repeat measurements in each gestational period. The RHI in pregnant women over 32 weeks of gestation was significantly lower than in those under 19 weeks of gestation. In contrast, plasma sFlt-1 levels in pregnant women over 32 weeks of gestation were significantly higher than those less than 20 weeks or between 20 and 31 weeks of gestation.

\section{RHI and Plasma sFlt-1 Levels in PE and Late PE Development Patients}

The RHI in PE patients was significantly higher than in normal pregnant women at the same gestational stage (31-36 weeks) (Figure 5A). On the other hand, plasma sFlt-1 levels showed over a 3-fold increase in PE patients $(p=0.004)$ (Figure 5B). RHI was measured in two PE patients after vaginal delivery. The RHI in one case was increased (1.58 to 1.86) nine 
Endothelial Function in Pregnancy

1 days after the delivery. In another case, it was similarly increased (1.65 to 1.84$)$ twelve days

2 after the delivery.

Three of 29 pregnant women developed PE in this study. The RHI and plasma sFlt-1

4 levels were measured at 10-15 weeks and 29-32 weeks of gestation, before the onset of PE in

5 these 3 patients. Both RHI and plasma sFlt-1 levels were no different at 10-15 weeks of

6 gestation. While RHI was still comparable at 29-32 weeks of gestation, plasma sFlt-1 levels

$7 \quad$ were slightly higher in patients with late PE development.

\section{COMMENT}

10 Salient findings of the present study were as follows: (1) Endothelial function evaluated by

11 PAT gradually deteriorated with the progression of gestational age in normal pregnant women.

12 (2) Plasma sFlt-1 levels exhibited a gradual increase late in pregnancy and were inversely

13 correlated with the RHI. (3) In the PE patients, RHI, an index of endothelial function, showed

14 a higher value in the presence of higher plasma sFlt-1 levels compared with normal pregnant women at similar gestational ages.

In the present study, we examined endothelial function by PAT in more detail and

17 demonstrated for the first time that RHI gradually decreases with increasing gestational age.

While some reports have claimed endothelial function ameliorates, others reported a decline 
Endothelial Function in Pregnancy

1 as a method of evaluating endothelial function. Lack of good reproducibility in the FMD

2 technique may explain, at least in part, inconsistent results in earlier studies. On the other

3 hand, the PAT technique provides more accurate information on endothelial function

4 regardless of the operator's skill and the autonomic nervous system activity of the examinee.

5 (15). Carty et al. has used PAT and reported that RHI at 28 weeks of normal pregnancy was

6 significantly lower than at 16 weeks (14). In the present study, we examined endothelial

7 function by PAT in more detail and demonstrated that RHI gradually decreases with

8 increasing gestational age, consistent with recent reports (14). Therefore we conclude that

9 endothelial function was deteriorating even in normal pregnancy.

Although it has been already shown that sFlt-1 levels are increasing during gestation, we

11 first that demonstrated that plasma sFlt-1 levels and RHI were inversely correlated. Until now,

12 there is no study showing a correlation between endothelial function and plasma sFlt-1levels

13 in normal pregnant women. As one of the reasons, we think that data analysis by FMD

14 method may have some difficulty, in terms of convenience, quantitation, and reproducibility.

15 Increased plasma sFlt-1 are suggested to neutralize VEGF and attenuate the beneficial effect

16 of VEGF on endothelial function in PE patients, though the relation between plasma sFlt-1

17 levels and endothelial function remains unclear in normal pregnancy. From the current results,

18 it can be speculated that the elevation of plasma sFlt-1 levels in the late pregnancy may cause

19 endothelial dysfunction. However, it is unknown whether elevated sFlt-1 influences 
Endothelial Function in Pregnancy

1 endothelial function directly and further studies will be needed to elucidate this relationship.

2 In our findings, the RHI in PE patients was relatively high. Markedly increased plasma

3 sFlt-1 levels in PE patients strongly suggest poorer endothelial function (22). It has already

4 been demonstrated that FMD is reduced in PE patients (23-25). Therefore our results appear

5 curious and must be interpreted with caution. The following two reasons may account for

6 about the cause of dissociation of this result. First, all PE patients in our study were medicated

7 with calcium blocker and/or magnesium sulfate, which potentially improved their endothelial

8 function. Second, there is a report that RH itself is slightly pressure-dependent $(\beta=0.007, p$

$9<0.001)$ (26). Generally, it is thought that endothelial dysfunction leads to hypertension and

10 arteriosclerosis. However, in the presence of high blood pressure, an evaluation of endothelial

11 function by PAT may be influenced by blood pressure. In PAT analysis, in contrast to our

12 result showing increased RHI, Yinon et al. (19) have reported decreased RHI in a study in PE

13 patients in which cases of relatively early onset were enrolled more frequently than in ours. It

14 is probable that markedly depressed endothelial function leads to a lower RHI despite the

15 presence of high blood pressure. Thus the relatively high RHI values in PE patients might be

16 due to their higher blood pressure level or to drugs, though this needs to be further elucidated.

19 function in the early stages of gestation is able to predict the onset of PE. 
Endothelial Function in Pregnancy

Angiogenic factors, including VEGF, in maternal vessels are important for maintaining

normal endothelial function. In PE patients, the normal balance between angiogenesis and

3 anti-angiogenesis has been compromised by circulating factors, including sFlt-1 and others,

4 from the placenta. Up to the present, there is no method to evaluate endothelial function that

5 combines non-invasiveness with convenience, quantitation and reproducibility. Although

6 further study must be done regarding the influence of blood pressure levels, it appears that

$7 \quad$ PAT will become a useful tool to assess maternal endothelial function hereafter. We believe

8 research into predicting or understanding the pathogenesis of PE might progress if more

9 measurements of anti-angiogenic or angiogenic factors in maternal blood can be added to the

10 accumulating data on this topic.

11 In conclusion, we here evaluated maternal endothelial function using the novel

12 methodology of the Endo-PAT2000 system and simultaneously measured plasma sFlt-1 levels

13 in pregnant women. Our investigation revealed that maternal endothelial function gradually

14 deteriorated while plasma sFlt-1 levels increased in inverse correlation with gestational age in

15 normal pregnancy. Anti-angiogenic or angiogenic factor profiling combining with RHI will

16 be valuable for predicting and understanding the pathogenesis of PE. 
Endothelial Function in Pregnancy

1 This work was supported by Grants-in-Aid for Scientific Research from the Ministry of

2 Education, Science, Culture and Sports, Japan (No. 21592096). The authors are grateful to

3 Mrs. Akiko Abe for secretarial and technical assistance, and deeply appreciate Prof. Minoru

4 Horie, Department of Cardiovascular and Respiratory Medicine, Shiga University of Medical

5 Science for providing us with the Endo-PAT2000 system.

6

\section{Declaration of Interest}

8 The authors report no conflict of interest. The authors alone are responsible for the content

9 and writing of the paper.

10

11 Figure Legends

12

13 Figure 1: Reactive hyperemia index (RHI) of the patients. A: RHI throughout the normal

14 gestation $(n=56)$. B: Comparison of RHI among 3 gestational periods $(\leq 19$ weeks, $20-31$ weeks, $\geq 32$ weeks) of normal pregnant women and non-pregnant women. $* *: p<0.001$

Figure 2: Plasma sFlt-1 levels of the patients. A: Plasma sFlt-1 levels throughout the normal gestation $(\mathrm{n}=56)$. B: Comparison of plasma sFlt-1 levels among normal pregnant women and non-pregnant women. a: $p<0.05$ vs. non-pregnant women, b: $p<0.05$ vs. $\leq 19$ weeks of gestation, c: $p<0.05$ vs. $20-31$ weeks of gestation. 
Endothelial Function in Pregnancy

2 Figure 3: Correlation of reactive hyperemia index to plasma sFlt-1 levels $(n=56)$.

$4 \quad$ Figure 4: The change of reactive hyperemia index (A) and plasma sFlt-1 levels (B) in serial 3

5 gestational periods in the same patients $(n=7)$.

6

7 Figure 5: Comparisons of reactive hyperemia index (A) and plasma sFlt-1 levels (B) between

$8 \quad$ PE $(n=7)$ and normal pregnant women $(n=17)$ at the same gestational age $(31-36$ weeks of 9 gestation).

10

\section{REFERENCES}

12 1. Robson SC, Hunter S, Boys RJ, et al. Serial study of factors influencing changes in cardiac output during human pregnancy. Am J Physiol 1989;256:H1060-1065.

2. Poston L. Maternal vascular function in pregnancy. J Hum Hypertens 1996;10:391394.

3. Roberts JM, Cooper DW. Pathogenesis and genetics of pre-eclampsia. Lancet 2001;357:53-56.

4. Dorup I, Skajaa K, Sorensen KE. Normal pregnancy is associated with enhanced 
Endothelial Function in Pregnancy

1

2 5. Faber-Swensson AP, O'Callaghan SP, Walters WA. Endothelial cell function

825. enhancement in a late normal human pregnancy. Aust N Z J Obstet Gynaecol 2004;44:525-529.

6. Quinton AE, Cook CM, Peek MJ. A longitudinal study using ultrasound to assess flow-mediated dilatation in normal human pregnancy. Hypertens Pregnancy 2007;26:273-281.

7. Savvidou MD, Kametas NA, Donald AE, et al. Non-invasive assessment of endothelial function in normal pregnancy. Ultrasound Obstet Gynecol 2000;15:502507.

8. Sierra-Laguado J, Garcia RG, Lopez-Jaramillo P. Flow-mediated dilatation of the brachial artery in pregnancy. Int J Gynaecol Obstet 2006;93:60-61.

9. Gokce N, Keaney Jr JF, Vita JA. Endotheliopathies: clinical manifestations of endothelial dysfunction. Thrombosis and Hemorrhage 1998:901-924.

10. Maynard SE, Min JY, Merchan J, et al. Excess placental soluble fms-like tyrosine kinase 1 (sFlt1) may contribute to endothelial dysfunction, hypertension, and proteinuria in preeclampsia. J Clin Invest 2003;111:649-658.

11. Koga K, Osuga Y, Yoshino O, et al. Elevated serum soluble vascular endothelial growth factor receptor 1 (sVEGFR-1) levels in women with preeclampsia. J Clin 
Endothelial Function in Pregnancy

Endocrinol Metab 2003;88:2348-2351.

12. Roberts JM, Taylor RN, Musci TJ, et al. Preeclampsia: an endothelial cell disorder. Am J Obstet Gynecol 1989;161:1200-1204.

13. Corretti MC, Anderson TJ, Benjamin EJ, et al. Guidelines for the ultrasound assessment of endothelial-dependent flow-mediated vasodilation of the brachial artery: a report of the international brachial artery reactivity task force. J Am Coll Cardiol 2002;39:257-265.

14. Carty DM, Anderson LA, Duncan CN, et al. Peripheral arterial tone: assessment of microcirculatory function in pregnancy. J Hypertens 2012;30:117-123.

15. Hamburg NM, Palmisano J, Larson MG, et al. Relation of brachial and digital measures of vascular function in the community: The Framingham Heart Study. Hypertension 2011;57:390-396.

16. Bonetti PO, Pumper GM, Higano ST, et al. Noninvasive identification of patients with early coronary atherosclerosis by assessment of digital reactive hyperemia. J Am Coll Cardiol 2004;44:2137-2141.

17. Nohria A, Gerhard-Herman M, Creager MA, et al. Role of nitric oxide in the regulation of digital pulse volume amplitude in humans. J Appl Physiol 2006;101:545548.

18. Aragones G, Ferre R, Girona J, et al. Small artery dilation and endothelial markers in 
Endothelial Function in Pregnancy

cardiovascular risk patients. Eur J Clin Invest 2012;42:34-41.

19. Yinon D, Lowenstein L, Suraya S, et al. Pre-Eclampsia is associated with sleepdisordered breathing and endothelial dysfunction. Eur Respir J 2006;27:328-333.

20. Bonetti PO, Barsness GW, Keelan PC, et al. Enhanced external counterpulsation improves endothelial function in patients with symptomatic coronary artery disease. $\mathrm{J}$ Am Coll Cardiol 2003;41:1761-1768.

21. ACOG practice bulletin. Diagnosis and management of preeclampsia and eclampsia. number 33, 2002. Obstet Gynecol 2002;99:159-167.

22. Sibai B, Dekker G, Kupferminc M. Pre-eclampsia. Lancet 2005;365:785-799.

23. Adali E, Kurdoglu M, Adali F, et al. The relationship between brachial artery flowmediated dilatation, high sensitivity c-reactive protein, and uterine artery doppler velocimetry in women with pre-eclampsia. J Clin Ultrasound 2011;39:191-197.

24. Chambers JC, Fusi L, Malik IS, et al. Association of maternal endothelial dysfunction with preeclampsia. JAMA 2001;285:1607-1612.

25. Yoshida A, Nakao S, Kobayashi M, et al. Flow-mediated vasodilation and plasma fibronectin levels in preeclampsia. Hypertension 2000;36:400-404.

26. Truschel E, Jarczok MN, Fischer JE, et al. High-throughput ambulatory assessment of digital reactive hyperemia: concurrent validity with known cardiovascular risk factors and potential confounding. Prev Med 2009;49:468-472. 
Table1: Clinical characteristics and pregnancy outcomes in study subjects.

\begin{tabular}{|c|c|c|c|c|}
\hline & $\begin{array}{c}\text { Non-pregnant } \\
\text { women }\end{array}$ & $\begin{array}{c}\text { Normal } \\
\text { pregnant } \\
\text { women }\end{array}$ & $\begin{array}{c}\text { Late PE } \\
\text { development }\end{array}$ & PE \\
\hline Subjects (n) & 9 & 26 & 3 & 7 \\
\hline Total number of measurement (n) & 9 & 56 & 6 & 7 \\
\hline Age (years) & $\begin{array}{l}32.0 \pm 2.4 \\
\quad(21-45)\end{array}$ & $\begin{array}{l}33.3 \pm 1.0 \\
(25-41)\end{array}$ & $\begin{array}{l}36.0 \pm 2.0 \\
\quad(34-40)\end{array}$ & $\begin{array}{l}34.6 \pm 0.6 \\
(24-36)\end{array}$ \\
\hline Primipara (n) & $(-)$ & $23 / 26$ & $1 / 3$ & $5 / 7$ \\
\hline \multicolumn{5}{|l|}{$\begin{array}{l}\text { Physical findings at the first time of } \\
\text { measurement: }\end{array}$} \\
\hline Body mass index $\left(\mathrm{kg} / \mathrm{m}^{2}\right)$ & $\begin{array}{l}19.3 \pm 0.5 \\
(16.6-21.4)\end{array}$ & $\begin{array}{c}24.1 \pm 0.9^{\mathrm{a}} \\
(18.4-37.9)\end{array}$ & $\begin{array}{l}21.7 \pm 1.4 \\
(18.8-23.1)\end{array}$ & $\begin{array}{l}26.4 \pm 1.4^{\mathrm{a}} \\
(23.1-32.0)\end{array}$ \\
\hline Systolic blood pressure $(\mathrm{mmHg})$ & $\begin{array}{l}110 \pm 2 \\
(98-120)\end{array}$ & $\begin{array}{l}108 \pm 2 \\
(90-133)\end{array}$ & $\begin{array}{c}108 \pm 6 \\
(100-119)\end{array}$ & $\begin{array}{c}136 \pm 5^{\mathrm{a}, \mathrm{b}} \\
(120-150)\end{array}$ \\
\hline Diastolic blood pressure $(\mathrm{mmHg})$ & $\begin{array}{l}66 \pm 3 \\
(52-75)\end{array}$ & $\begin{array}{l}63 \pm 2 \\
(46-74)\end{array}$ & $\begin{array}{l}65 \pm 4 \\
(60-72)\end{array}$ & $\begin{array}{l}87 \pm 3^{\mathrm{a}, \mathrm{b}, \mathrm{c}} \\
\quad(76-96)\end{array}$ \\
\hline \multicolumn{5}{|l|}{ Outcomes: } \\
\hline Gestational age at delivery (weeks) & $(-)$ & $\begin{array}{c}39 \\
{[38-40]}\end{array}$ & $\begin{array}{c}37 \\
{[37-37.5]}\end{array}$ & $\begin{array}{c}35^{\mathrm{b}} \\
{[34-37]}\end{array}$ \\
\hline Neoatal weight (g) & $(-)$ & $\begin{array}{c}2986 \\
{[2852-3078]}\end{array}$ & $\begin{array}{c}2866 \\
{[2643-2998]}\end{array}$ & $\begin{array}{c}1986^{\mathrm{b}, \mathrm{c}} \\
{[1805-2437]}\end{array}$ \\
\hline SGA (n) & $(-)$ & $0 / 26$ & $0 / 3$ & $2 / 7$ \\
\hline
\end{tabular}

PE: preeclampsia, SGA: small for gestational age. Values are the mean \pm SEM and (range) or median value with [interquartile range]. a: $p<0.05$ vs. non-pregnant women, b: $p<0.05$ vs. normal pregnant women, c: $p<0.05$ vs. late PE development 

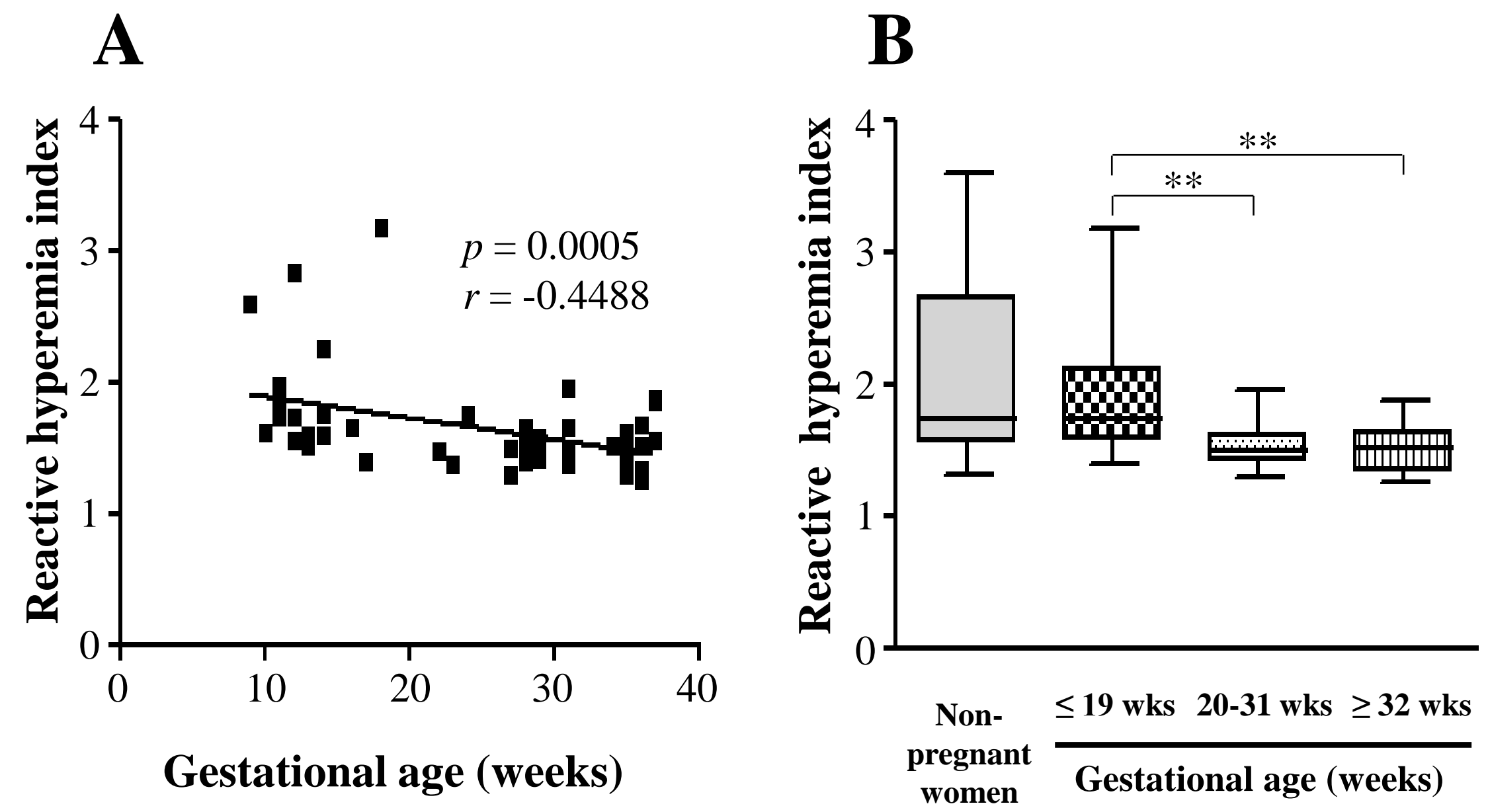

(Number)

(9)

(18)

(21)

(17) 

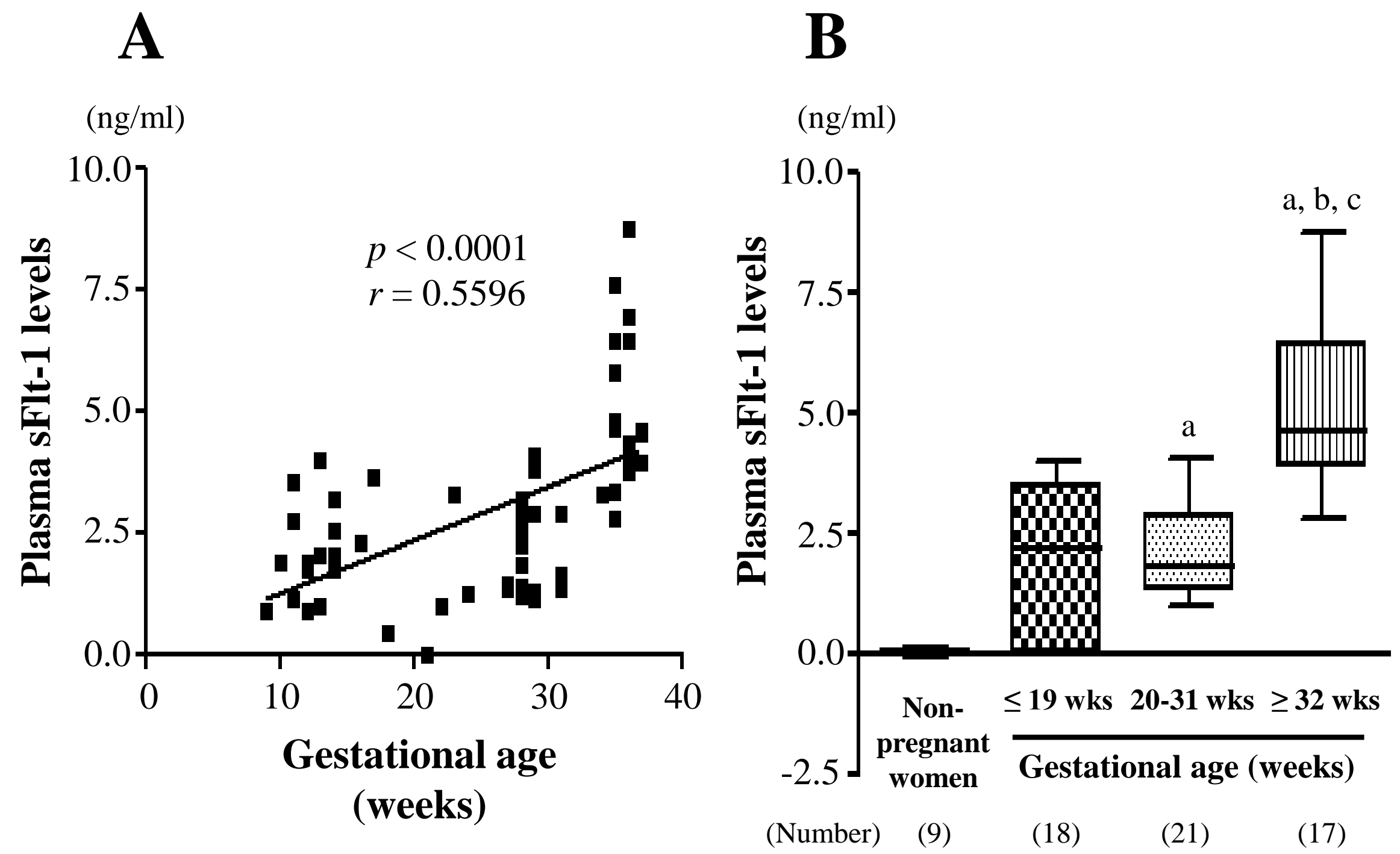


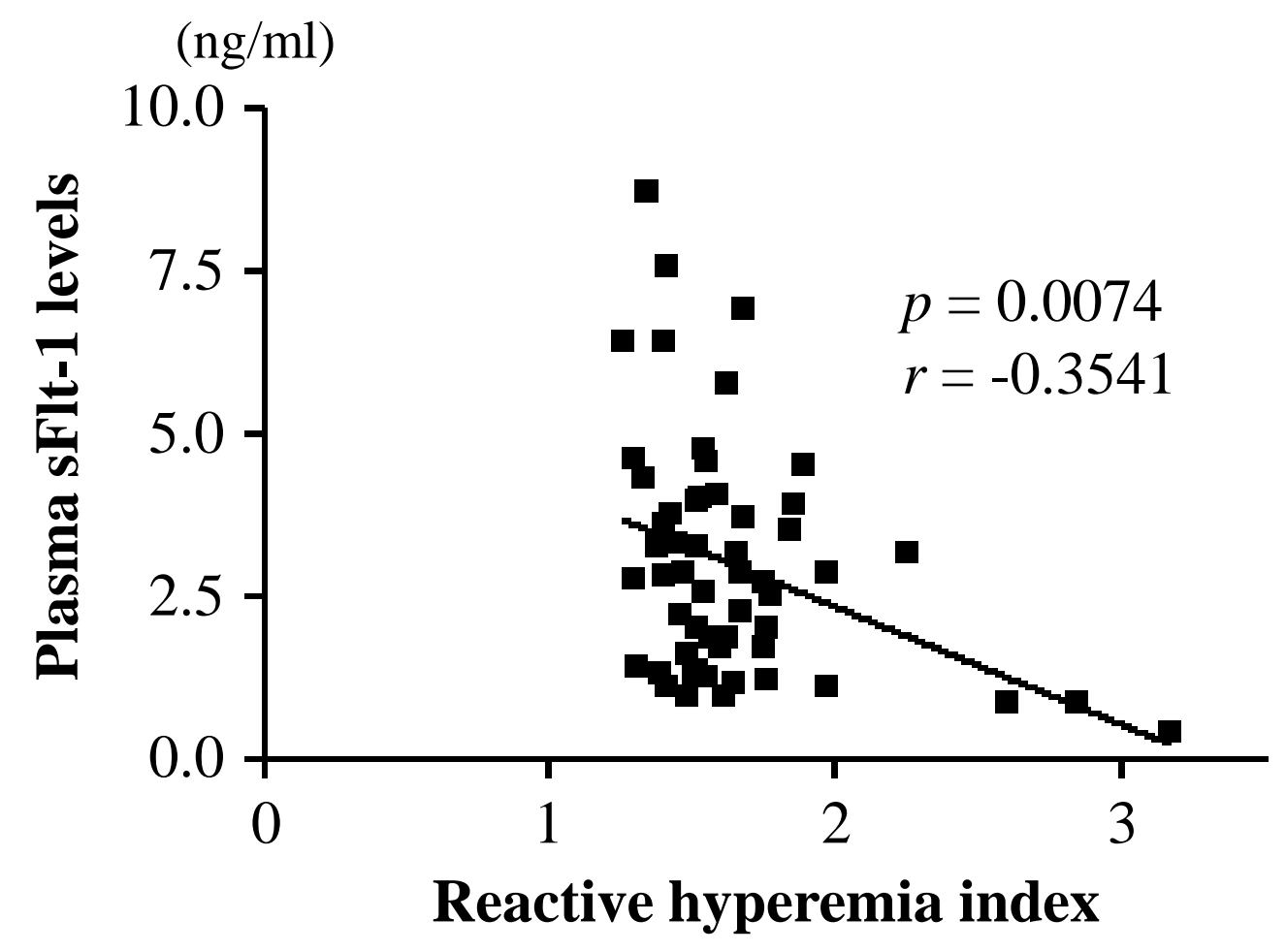



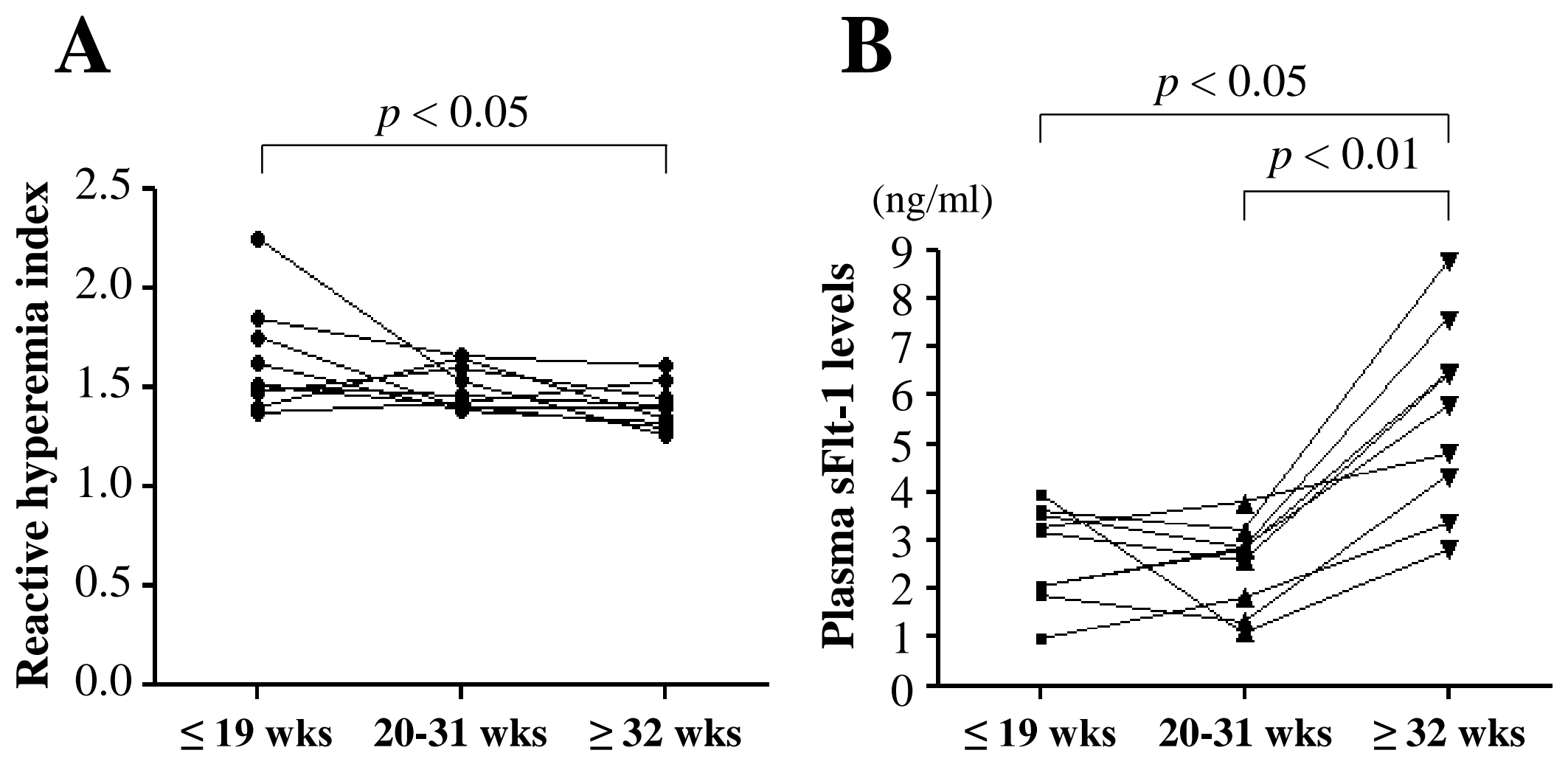


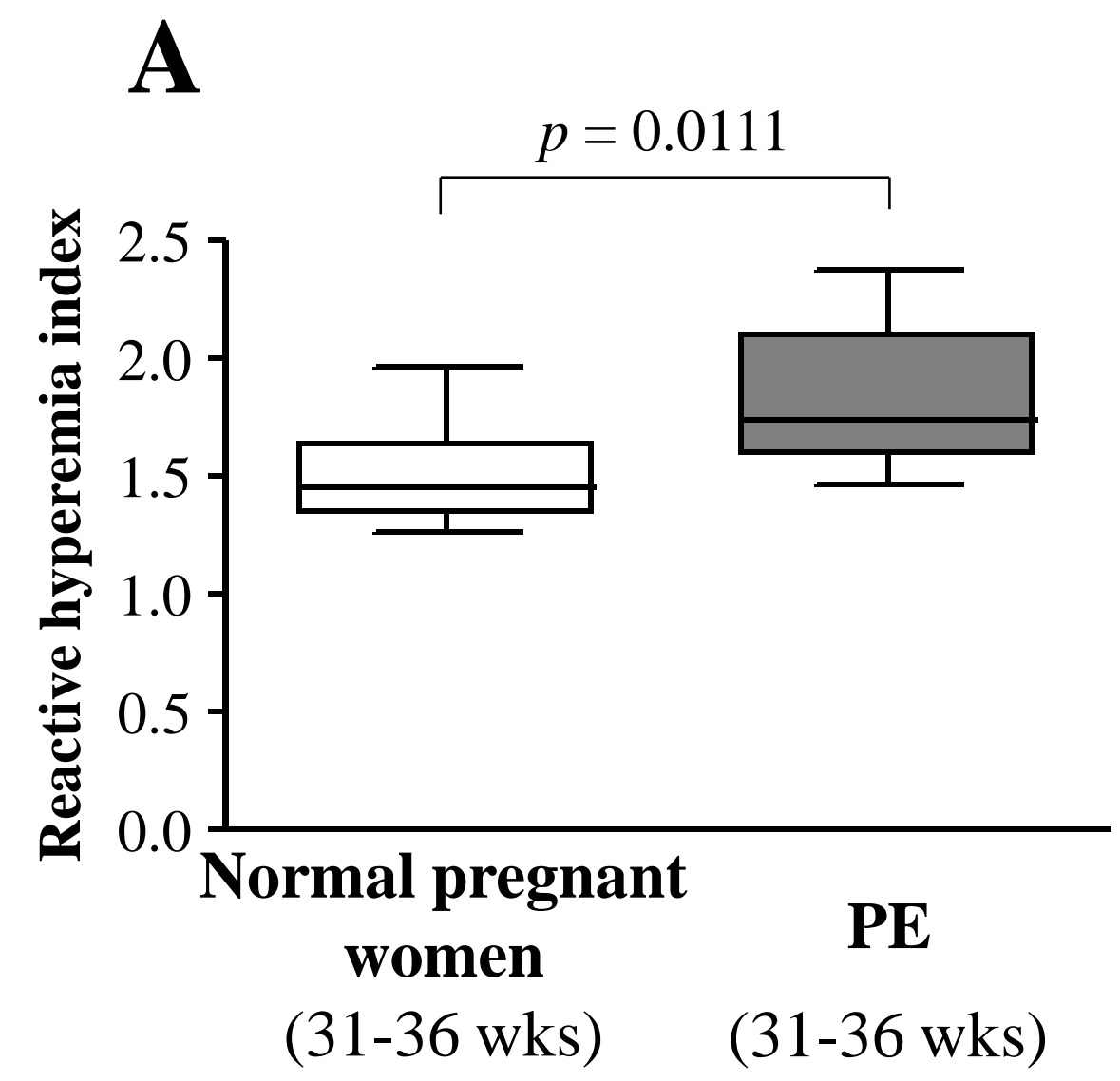

B

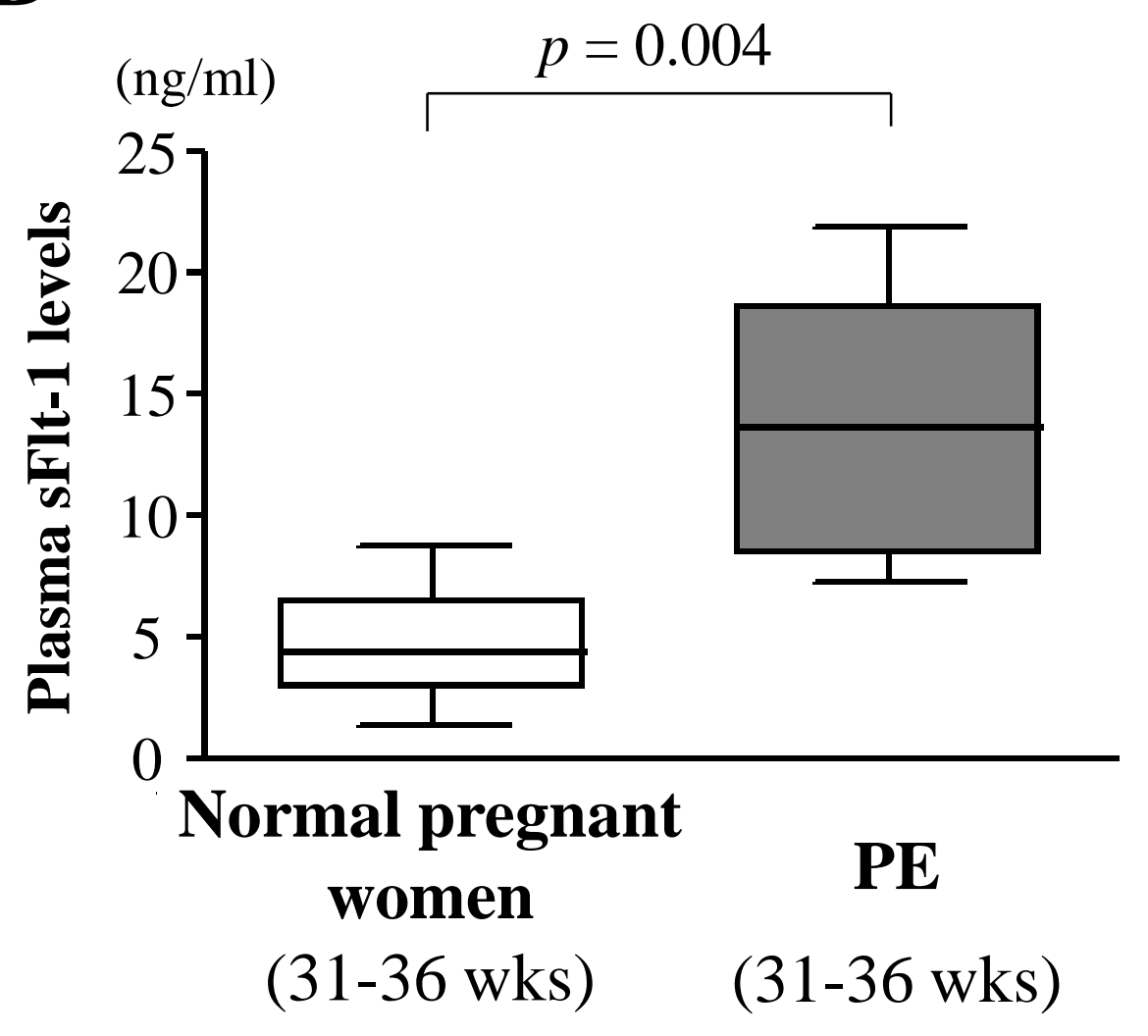

